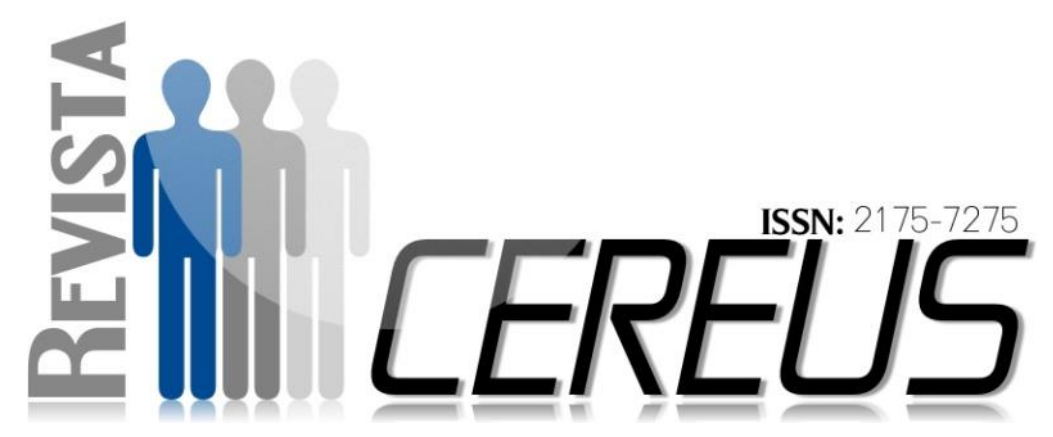

\title{
RELAÇÃO ENTRE ENTEROPARASITOSES E RENDIMENTO ESCOLAR EM ALUNOS DO ENSINO FUNDAMENTAL DE GURUPI, TOCANTINS
}

\author{
SILVA, Marcos Gontijo da ${ }^{1}$, \\ MELO, Marllos Peres de, ${ }^{2}$, \\ GONTIJO, Érica Eugênio Lourenço ${ }^{3}$, \\ NETO, João Bartholomeu 4 , \\ SILVEIRA, Janne Marques ${ }^{5}$
}

\section{RESUMO}

INTRODUÇÃO: A busca por uma escola inclusiva, de qualidade para todos, têm avançado com o apoio dos governos e sociedade civil, que claramente se posicionam pela redução do analfabetismo. OBJETIVO: o presente trabalho busca demonstrar a influência das parasitoses intestinais no desempenho escolar em estudantes do ensino fundamental público do município de Gurupi, Tocantins, ${ }^{1}$ Doutor em medicina tropical e saúde pública. Professor titular do Centro Universitário Unirg

${ }^{2}$ Doutor em agronomia pela UFT. Professor titular do Centro Universitário Unirg

${ }^{3}$ Doutora em Ciências da Saúde pela UFG. Professora do Centro Universitário Unirg

${ }^{4}$ Mestre em educação física. Professor do Centro Universitário Unirg

${ }^{5}$ Mestre em fisiologia. Professora do Centro Universitário Unirg 
Brasil. METODOLOGIA: Foram coletadas, em domicilio, amostras de fezes de 205 alunos, de 5 escolas públicas da zona urbana da Cidade de Gurupi, Tocantins, Brasil, com idades entre cinco e 12 anos. As amostras foram analisadas por três métodos analíticos cada: método de Faust, para verificar a presença de cistos de protozoários e ovos leves de helmintos; método de Hoffman, para ovos pesados; e método Rugai para verificar a presença de larvas. A amostra foi dividida em dois grupos: Grupo caso, formado por 87 crianças infectadas e o grupo controle, formado por 118 crianças não contaminadas. O desempenho escolar foi calculado pelas médias das notas dos alunos nas disciplinas de língua portuguesa, matemática e ciências durante um ano (quatro bimestres). Verificou-se a relação entre o poliparasitismo e o desempenho escolar. Buscou-se a influência e o impacto de cada parasitos/comensais e helmintos no desempenho escolar. RESULTADO: Das 205 amostras avaliadas, 42,93\% apresentaram resultado positivo ao exame parasitológico para um ou mais parasitos/comensais. Quanto ao rendimento escolar $14.15 \%$ apresentaram rendimento insuficiente e $85.85 \%$ rendimento suficiente.

Palavras chave: Parasitoses; Interdisciplinaridade; desempenho escolar.

\section{RELATIONSHIP BETWEEN ENTEROPARASITOSES AND SCHOOL YIELD IN STUDENTS OF THE FUNDAMENTAL TEACHING OF GURUPI, TOCANTINS.}

\section{ABSTRACT}

INTRODUCTION: The quest for an inclusive, quality school for all has advanced with the support of governments and civil society, which are clearly positioned to reduce illiteracy. PURPOSE: the present work aims to demonstrate the influence of intestinal parasitoses on school performance in students of public primary education in the municipality of 
Gurupi, Tocantins, Brazil. METHODS: Feces samples of 205 students from 5 public schools in the urban area of the city of Gurupi, Tocantins, Brazil, aged 5 to 12 years, were collected at home. The samples were analyzed by three analytical methods each: Faust method, to verify the presence of protozoan cysts and light helminth eggs; Hoffman's method for heavy eggs; and Rugai method to verify the presence of larvae. The sample was divided into two groups: a case group composed of 87 infected children and the control group of 118 uncontaminated children. The school performance was calculated by the means of the students' grades in Portuguese, mathematics and science subjects during one year (four months). The relationship between polyparasitism and school performance was verified. The influence and impact of each parasite / helminth parasite on school performance was sought. RESULTS: Of the 205 samples evaluated, $42.93 \%$ presented a positive result on parasitological examination for one or more parasites / commensals. Regarding school performance, $14.15 \%$ had insufficient income and $85.85 \%$ had sufficient income..

Keywords: Parasitoses; Interdisciplinarity; school performance. 


\section{NTRODUÇÃO}

A busca por uma escola inclusiva, de qualidade para todos, têm avançado com o apoio dos governos e sociedade civil, que claramente se posicionam pela redução do analfabetismo (BRASIL, 2016). A educação no Brasil é um direito de assegurado a todos pela Constituição Federal de 1988 (BRASIL, 2012). Dentre as diversas metas do PNE 2014-2024, destaca-se que a meta 5 visa alfabetizar todas as crianças, no máximo, até o final do $3^{\circ}$ (terceiro) ano do ensino fundamental (BRASIL, 2014).

Para o desenvolvimento escolar integral das crianças é fundamental além do acesso à escola, de processos pedagógicos modernos e eficientes, que as crianças tenham a sua saúde plena, visto que qualquer debilidade física pode comprometer 0 desempenho nos anos iniciais da sua escolarização (BRASIL, 2016).

Segundo MENDES (2012), os fatores correlacionados com o fracasso escolar são classificados em extraescolares e intra-escolares. Os intraescolares estão relacionados ao trabalho desenvolvido pelos professores e especialistas, as avaliações do desempenho dos alunos, currículo e os programas. Os extraescolares, estão associados às más condições de vida e subsistência, as dificuldades econômicas, responsáveis dentre outros fatores pela fome e desnutrição, a falta de moradias adequadas e de saneamento e as enteroparasitoses que convivem as classes sociais menos privilegiadas (MACHADO et al., 2013).

As crianças são mais susceptíveis a doenças infectoparasitárias devido aos hábitos higiênicos inadequados e sua pouca eficiência imunológica. As enteroparasitoses afetam negativamente o desempenho escolar, pois prejudica o desenvolvimento assim como limitam suas potencialidades individuais (MONTEIRO et al., 2009; MAIA; HASSUM, 2011; ANTUNES; LIBARDONI, 2017; COSTA et al., 2017).

Os parasitas intestinais estão entre os patógenos mais frequentemente encontrados em seres humanos (PINHEIRO, 2011). As parasitoses intestinais podem causar danos ao organismo e comprometer o desempenho de atividades físicas e intelectuais de crianças, refletindo em 
menor capacidade de atenção, aprendizado e rendimento escolar (CLERICI; PIGATTO, 2016; PIRES et al., 2016; SILVA et al., 2016; MONTEIRO, 2017). Nessa perspectiva,

\section{MÉTODO}

Entre fevereiro de 2007 a dezembro de 2010, foram coletadas, em domicilio, amostras de fezes de 205 alunos, de 5 escolas públicas da zona urbana da Cidade de Gurupi, Tocantins, Brasil, com idades entre cinco e 12 anos. Foram coletadas três amostras por aluno em dias alternados.

As amostras foram analisadas por três métodos analíticos cada: método de Faust, para verificar a presença de cistos de protozoários e ovos leves de helmintos; método de Hoffman, para ovos pesados; e método Rugai para verificar a presença de larvas.

Os responsáveis dos alunos participantes autorizaram por escrito a inclusão dos seus filhos na pesquisa. Foram pesquisados alunos das escolas (Escola Municipal Rui Barbosa, Escola Municipal Odair Lucio, Escola Municipal Orlindo Pereira, Escola Espírita Bezerra de Menezes, Escola Municipal Agripimo de Sousa Galvão e Alunos da Escola Municipal Antônio de Almeida o presente trabalho busca demonstrar a influência das parasitoses intestinais no desempenho escolar em estudantes do ensino fundamental público do município de Gurupi, Tocantins, Brasil.

Veras). Esta pesquisa foi aprovada pelo comitê de ética e pesquisa do centro universitário UNIRG com certificado nํㅜ 0104/2010.

Das 205 amostras avaliadas, $42,92 \%$ apresentaram resultado positivo ao exame parasitológico para um ou mais parasitos/comensais. A amostra foi dividida em dois grupos: Grupo caso, formado por 87 crianças infectadas e o grupo controle, formado por 118 crianças não contaminadas.

O desempenho escolar foi calculado pelas médias das notas dos alunos nas disciplinas de língua portuguesa, matemática e ciências durante um ano (quatro bimestres). Foram descartadas as notas de educação física, ensino religioso e artes. As notas obtidas foram convertidas em 2 conceitos, sendo, notas superiores ou igual a 6 "suficiente" e menor que 6 "insuficiente". 
Foi realizada a correlação entre a presença de enteroparasitos e o rendimento escolar.

Verificou-se a relação entre o poliparasitismo e 0 desempenho escolar. Buscou-se a influência e o impacto de cada parasitos/comensais e helmintos no desempenho escolar.

\section{RESULTADOS E DISCUSSÃO}

Das 205 amostras avaliadas, $42,93 \%$ apresentaram resultado positivo ao exame parasitológico para um ou mais parasitos/comensais sendo que $18,53 \%$ estudantes tinham Giardia lamblia, 16,09\% Entamoeba coli, 3,90\% contaminadas por Entamoeba histolytica e Himenolepis nana, 1,95\% com Enterobius vermicularis, Ascaris lumbricoides e Ancilostomídeos, 0,97\% com Endolimax nana e $0,48 \%$ com Trichuris trichiura e Taenia spp.

Quanto ao rendimento escolar $14.15 \%$ apresentaram rendimento insuficiente e $85.85 \%$ rendimento suficiente.Observa-se que a presença de enteroparasitos influencia negativamente no rendimento escolar. Os enteroparasitos aumentam em 3.2 vezes a chance dos alunos terem notas insuficientes, sendo que os
A avaliação da correlação foi feita usando o teste ODSS RATIO (OD) onde foi construído tabelas $2 \times 2$ avaliando diferença no rendimento escolar em dois grupos um infectado com enteroparasitos e outros não infectados. Foi considerado valor de $p \leq 5 \%$.

protozoários aumentam 2.96 vezes e os helmintos 4.41 vezes (Tabela 1 ).

As doenças parasitárias estão associadas com a desnutrição e diarreia crônica, prejudica o desenvolvimento físico e mental, reflete no rendimento escolar com redução da capacidade física e intelectual das crianças parasitadas, suprimir 0 sistema imunológico e é causa de morbimortalidade elevada (MAIA; HASSUM, 2011; CLERICI; PIGATTO, 2016; SILVA et al., 2016).

Os resultados deletérios das parasitoses são explicados redução das reservas energéticas disponíveis nos indivíduos. Dessa forma, diminui sua capacidade física e mental, sua motivação, prejudicando sua interação social (PIRES et al., 2016). 
Tabela 1: Correlação entre enteroparasitoses e rendimento escolar em alunos do ensino fundamental de Gurupi, Tocantins, Brasil

\begin{tabular}{|c|c|c|c|c|c|c|c|}
\hline \multirow[t]{2}{*}{ 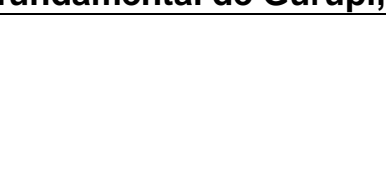 } & \multicolumn{2}{|c|}{$\begin{array}{l}\text { Rendimento } \\
\text { escolar } \\
\text { Suficiente }\end{array}$} & \multicolumn{2}{|c|}{$\begin{array}{l}\text { Rendimento } \\
\text { escolar } \\
\text { Insuficiente }\end{array}$} & \multirow[t]{2}{*}{ OR } & \multirow[t]{2}{*}{ IC 95\% } & \multirow[t]{2}{*}{$\begin{array}{l}\text { Valor } \\
\text { de } p\end{array}$} \\
\hline & n. & $\%$ & n. & $\%$ & & & \\
\hline $\begin{array}{l}\text { INFECÇÃO POR } \\
\text { ENTEROPARASITOS }\end{array}$ & 176 & & 29 & & & & \\
\hline $\begin{array}{l}\text { Sim } \\
\text { Não }\end{array}$ & $\begin{array}{c}63 \\
113\end{array}$ & $\begin{array}{l}35.80 \% \\
64.20 \%\end{array}$ & $\begin{array}{l}18 \\
11\end{array}$ & $\begin{array}{l}62.07 \% \\
37.93 \%\end{array}$ & $\begin{array}{c}2.93 \\
1\end{array}$ & $1.30-6.60$ & 0.007 \\
\hline \multicolumn{8}{|l|}{ POLIPARASITADOS } \\
\hline $\begin{array}{l}\text { Sim } \\
\text { Não }\end{array}$ & $\begin{array}{c}13 \\
163\end{array}$ & $\begin{array}{l}7.39 \% \\
92.61 \%\end{array}$ & $\begin{array}{c}2 \\
27\end{array}$ & $\begin{array}{c}6.90 \% \\
93.10 \% \\
\end{array}$ & $\begin{array}{c}1.08 \\
1\end{array}$ & $0.23-5.04$ & 0.920 \\
\hline \multicolumn{8}{|l|}{$\begin{array}{l}\text { PRESENÇA DE } \\
\text { HELMINTOS }\end{array}$} \\
\hline 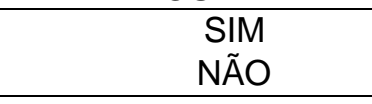 & $\begin{array}{c}14 \\
162 \\
\end{array}$ & $\begin{array}{l}7.95 \% \\
92.95 \% \\
\end{array}$ & $\begin{array}{c}6 \\
23 \\
\end{array}$ & $\begin{array}{l}20.69 \% \\
79.31 \% \\
\end{array}$ & $\begin{array}{c}3.02 \\
1\end{array}$ & $1.05-8.64$ & 0.032 \\
\hline \multicolumn{8}{|l|}{$\begin{array}{l}\text { PRESENÇA DE } \\
\text { PROTOZOÁRIOS }\end{array}$} \\
\hline $\begin{array}{l}\text { SIM } \\
\text { NÃO }\end{array}$ & $\begin{array}{c}57 \\
119\end{array}$ & $\begin{array}{l}32.39 \% \\
67.61 \%\end{array}$ & $\begin{array}{l}12 \\
17\end{array}$ & $\begin{array}{l}41.38 \% \\
58.62 \%\end{array}$ & $\begin{array}{c}1.47 \\
1\end{array}$ & $0.66-3.29$ & 0.342 \\
\hline
\end{tabular}

As enteroparasitoses tem potencial de interferir na qualidade de vida dos parasitados de classes sociais mais baixas, em situação de desnutrição e possuem maior prevalência em locais de fácil disseminação dos parasitas intestinais tais como escolas e creches (MONTEIRO et al., 2009; SOUZA et al., 2015; PIRES et al., 2016; SILVA et al., 2017).

Essas doenças parasitárias possuem capacidade de influenciar o estudo nutricional, crescimento e capacidade cognitiva das crianças em idade escolar. O processo inflamatório desencadeado pelas ações mecânicas, químicas e antigênicas dos parasitas intestinais podem afetar as funções e estrutura do trato gastrintestinal, alterando os mecanismos de absorção e digestão dos alimentos (PIRES et al., 2016).

Os quadros de desnutrição em crianças leva à redução da capacidade cognitiva, maior susceptibilidade a doenças infecciosas, diminuição da absorção intestinal e do metabolismo (PINHEIRO, 2011; MACHADO et al., 2013; ANTUNES; LIBARDONI, 2017; LACERDA; JARDIM, 2017).

A associação das enteroparasitoses e rendimento escolar na infância vem sendo descrita por diversos estudos, que corroboram com o fato das parasitoses promovem uma 
redução da capacidade cognitiva nas crianças devido ao déficit nutricional (MONTEIRO et al., 2009; PINHEIRO, 2011; CLERICI; PIGATTO, 2016; SILVA et al., 2016; MONTEIRO, 2017). O fracasso escolar no Brasil é um problema histórico e comum em todos os níveis de ensino, porém com maior incidência nos primeiros anos da escolarização (CLERICl; PIGATTO, 2016).

A precária alimentação na infância em conjunto com as condições de saneamento básico, torna-se comum a ocorrência de enteroparasitoses, expondo crianças a uma situação de desequilíbrio crônico, principalmente pela carência de ferro (MAIA; HASSUM, 2011). Comprometendo o comportamento do aluno, principalmente em relação à capacidade de concentração, atenção e rendimento escolar, reduzindo a capacidade para o aprendizado escolar (MONTEIRO, 2017).

Crianças em idade escolar, em especial as que vivem em condições sociais precárias, são mais contaminadas e de forma bem mais grave pelas enteroparasitoses, pois sua capacidade imunológica é deficitária por motivos carenciais para a eliminação dos agentes infecciosos intestinais (PIRES et al., 2016).

$\mathrm{Na}$ fase escolar, a presença destes consistem em um fator agravante da subnutrição, levando à morbidade nutricional, que é, em geral, acompanhada da diarreia crônica, fraqueza, náuseas, sendo assim fatores que refletirão diretamente no rendimento escolar, (MENDES, 2012; MACHADO et al., 2013; SOUZA et al., 2015; CLERICI; PIGATTO, 2016; PIRES et al., 2016).

Quanto a espécie de parasito que influencia deleteriamente no rendimento escolar no trabalho, a Giardia lamblia aumentou em 4.91 vezes a chance dos alunos terem rendimento insuficiente e o Ascaris lumbricoides 10.04 vezes.

Segundo MONTEIRO et al., (2009), a presença de anemia e desnutrição associada

a enteroparasitoses é resultante da ação espoliadora (Ascaris lumbricóides), do atapetamento e má absorção intestinal (Giardia lamblia), da ação hematofágica (Ancylostoma sp.) e da ulceração das mucosas intestinais (Entamoeba histolytica), podendo desencadear constantes hemorragias intestinais no indivíduo. O quadro clínico depende da carga parasitária, 
idade, estado nutricional e fisiológico do organismo, bem como da co-infecção entre espécies parasitárias patogênicas. Nesse contexto a desnutrição e anemia em escolares influenciam o comportamento, em especial quanto à capacidade de atenção e rendimento escolar, reduz a habilidade e disposição para o aprendizado, e em decorrência disso proporciona repetência, idade inadequada da criança na série e evasão escolar (VASCONCELOS et al., 2016).

A má absorção de nutrientes, perda de sangue, diarreia, capacidade diminuída de trabalho, reduzida taxa de crescimento devido às cargas parasitarias constituem um problema grave sanitário e social (PINHEIRO, 2011; MENDES, 2012; OLIVEIRA, 2013; BARBOSA, 2015; MONTEIRO, 2017).Incontestável assim, a relação que há entre verminoses e 0 baixo rendimento dos alunos, que tem seu desenvolvimento físico e cognitivo

\section{CONCLUSÃO}

As enteroparasitoses possuem relação direta com o baixo rendimento escolar no grupo estudado. As principais enteroparasitoses que prejudicam o impactado, refletindo em altas taxas de evasão e fracasso escolar.Crianças desnutridas e com infecção por Ascaris lumbricoides e Giardia lamblia apresentam níveis mais baixos de aprendizado que crianças sadias (MONTEIRO et al., 2009; BELO et al., 2012; PIRES et al., 2016; ANTUNES; LIBARDONI, 2017; MONTEIRO, 2017).

Os resultados evidenciam que as enteroparasitoses permanecem como grave problema social, vitimando crianças e devido a dificuldades de aprendizado escolar, que repercute no desempenho estudantil, assim como os demais aspectos sociais da vida destes estudantes.

O presente estudo permite observar claramente a associação entre as enteroparasitoses e rendimento escolar. Dentre as principais limitações do estudo destaca-se a ausência de exame de sangue e avaliação corporal para estabelecer a relação entre os fatores nutricionais e desempenho escolar. aprendizado são a ascaridíase e a giardíase.

É possível afirmar que o garantindo acesso das crianças à escola não 
garante igualdade de acesso ao favorecem o fracasso escolar em aprendizado, visto que estas doenças crianças.

Tabela 2: Correlação entre as principais espécies de parasitos encontradas e o rendimento escolar em alunos do ensino fundamental de Gurupi, Tocantins, Brasil

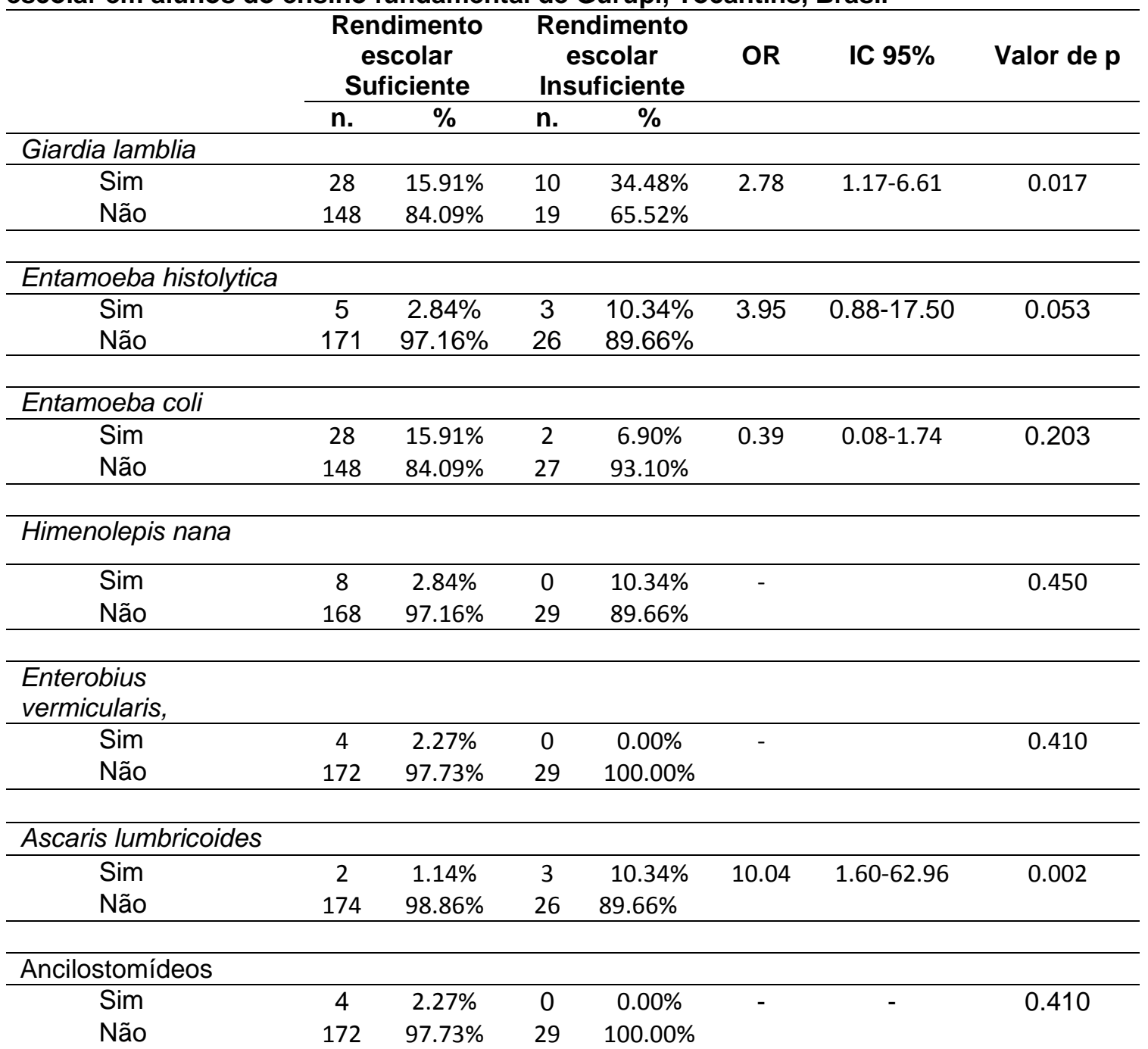

$\mathrm{n} .=$ Frequência Absoluta, $\%=$ Frequência relativa, $\mathrm{OR}=$ Odds Ratio, IC = Intervalo de confiança, $\mathrm{p}=$ Valor de $\mathrm{p}$

\section{REFERÊNCIAS}

ANTUNES, A. S.; LIBARDONI, K. S. D. B. Prevalence of enteroparasitosis in children of day-care center in the municipality of Santo Ângelo, RS. Revista Contexto \& Saúde, v. 17, n. 32, p. 144-156, 2017. 
BARBOSA, A. D. A. L. Plano de intervenção para redução da prevalência de parasitoses intestinais nos escolares da ESF do povoado de cana brava, zona rural no município de São Sebastião - AL. 2015. 27 f. Universidade Federal de Minas Gerais. 2015.

BELO, V. S.; OLIVEIRA, R. B. DE; FERNANDES, P. C.; et al. Fatores associados à ocorrência de parasitoses intestinais em uma população de crianças e adolescentes.

Revista Paulista de Pediatria, v. 30, n. 2, p. 195-201, 2012.

BRASIL. Constituição da República Federativa do Brasil: texto constitucional promulgado em 5 de outubro de 1988, com as alterações adotadas pelas Emendas Constitucionais nos 1/1992 a 68/2011, pelo Decreto Legislativo no 186/2008 e pelas Emendas Constitucionais. Brasilia, 2012.

BRASIL. Planejando a Próxima Década Conhecendo as 20 Metas do Plano Nacional de Educação. Secretaria de Articulação com os Sistemas de Ensino (MEC/ SASE), 2014.

BRASIL. Relatório educação para todos no Brasil 2000-2015. Ministério da Educação - Brasília: MEC, 2016.

CLERICI, D. J.; PIGATTO, A. G. S. Association between intestinal parasitosis and school performance: a systematic review. Disciplinarum Scientia | Saúde, v. 16, n. 1, p. 1-10, 2016.

COSTA, A. F. N. DA; MORAIS, J. O.; MELO, J. A. DE; SANTOS, E. A. DOS; MORAIS5, A. B. DE. Prevalence of enteroparasitosis in children of a public childcare. Temas em Saúde, v. 17, n. 1, p. 66-80, 2017.

LACERDA, J. DA S.; JARDIM, C. M. L. Estudo da prevalência de parasitoses intestinais em pacientes de um laboratório privado de Araçatuba-SP. Revista Saúde UniToledo, v. 1, n. 1, p. 107-120, 2017.

MACHADO, E. R.; CAMPOS, R. R.; NASCIMENTO, V. V. DO. Enteroparasitoses entre escolares da cidade de Águas Lindas de Goiás. Ensaios e Ciência: Ciências Biológicas, Agrárias e da Saúde, v. 17, n. 5, p. 19-32, 2013.

MAIA, C. V. DE A.; HASSUM, I. C. Intestinal parasites and social-sanitary aspects in northeastern Brazil in the twenty-first century: a literature review. Revista Brasileira de Geografia Médica e da Saúde, v. 12, n. 23, p. 20-30, 2011.

MENDES, A. D. A. R. Saúde escolar e educação integral: a relação entre as parasitoses intestinais e o desempenho escolar do aluno da escola municipal de 
ensino fundamental Roberto Turbay em Ariquemes-RO. 2012. 0-128 f. Universidade Federal de Rondônia. 2012.

MONTEIRO, A. C. DA S. Prevalência e fatores associados à enteroparasitoses em escolares. 2017. 82 f. UNIVERSIDADE FEDERAL DA PARAÍBA. 2017.

MONTEIRO, A. M. DE C.; SILVA, E. F. DA; ALMEIDA, K. DE S.; et al. Parasitoses intestinais em crianças de creches públicas localizadas em bairros periféricos do munícipio de Coari, Amazonas, Brasil. Revista de Patologia Tropical, v. 38, n. 4, p. 284-290, 2009.

OLIVEIRA, J. L. L. DE. Parasitoses intestinais: o ensino como ferramenta principal na minimização destas patologias. 2013. 76 f. Centro Universitário de Volta Redonda. 2013.

PINHEIRO, P. LOPES. Enteroparasitoses na infância, seus determinantes sociais e principais consequências, seus determinantes sociais e principais consequências: Uma revisão bibliográfica. 2011. 22 f. Universidade Federal de Minas Gerais. 2011.

PIRES, E. DA C. R.; GUIMARÃES, F. P.; DINIZ, J. C.; FROESELER, M. V. G.; MATA, L. C. C. DA. Abordagem interdisciplinar das parasitoses intestinais em escolares da microrregião de Sete Lagoas-MG. Arquivos de Ciências da Saúde da UNIPAR, v. 2, n. 31, p. 111-116, 2016.

SILVA, T. B. DA; CARDOSO, T. DOS R.; MARQUEZ, D. D. S.; et al. Prevalência de enteroparasitoses em casos diagnosticados no município de João Pinheiro-MG. Psicologia e Saúde em Debate, v. 2, n. 1, p. 18-29, 2016.

SILVA, V. G. DA; SANTOS, G. C. DOS; FERREIRA, V. M. DA S. Enteroparasitas veiculados em folhas de alfaces (Lactuca sativa) comercializadas na feira livre da cidade de Governador Valadares, Minas Gerais. Enciclopédia Biofera, v. 14, n. 25, p. 1343-1352, 2017.

SOUZA, M. P.; THOME, M. P. M.; SOUZA, Á. D. Enteroparasitoses em alunos da escola municipal antonio ferreira da fonseca, localidade rural do município de Italva (RJ). Reinpec, v. 1, n. 2, p. 127-135, 2015.

VASCONCELOS, C. S.; ALMEIDA, M. B. DE; BRITO, R. G. DE; et al. Enteroparasitoses humanas em Aracaju, SE. RBAC, v. 48, n. 4, p. 356-362, 2016. 